\title{
El Puntapié Inesperado Preguntas sobre el futuro de las Escuelas de Negocios en Chile
}

Juan Pablo Subiabre PhD (c) en Management Universidad de Bristol, Reino Unido
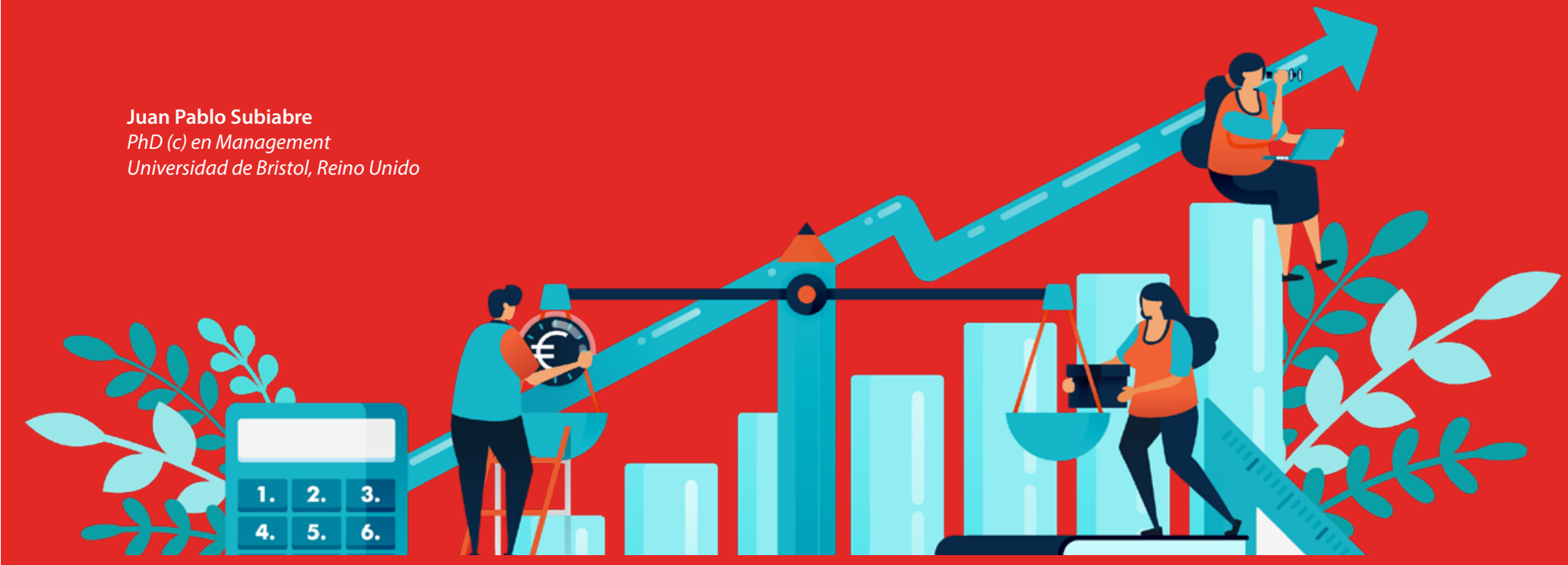

En el presente artículo planteo algunas inquietudes sobre el futuro de las escuelas de negocios en Chile. Estas interrogantes nacen a raíz de la actual crisis sanitaria y la transformación que esta ha desencadenado en las relaciones laborales y en el mundo organizacional. Para ello, comenzaré explicando los efectos que el impasse relevancia-rigor ha tenido en el modelo de desarrollo adoptado por las escuelas de negocios en las últimas décadas. A continuación, planteo que, si bien la adopción de este modelo reportó enormes beneficios en el pasado, hoy en día resulta un impedimento para adaptarse de forma ágil a un entorno impredecible. Finalmente, sugiero que la pandemia debiera catalizar una serie de cambios en la forma en que las escuelas de negocios entienden su relación con lo humano.

\section{Relevancia y Rigor}

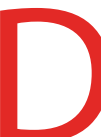
esde su creación hace menos de dos siglos, las escuelas de negocios nunca han recibido ni el reconocimiento ni la validación que han deseado. Este anhelo frustrado es comúnmente referido como "envidia de la física" (Bennis \& O’Toole,
2005), es decir que, desde sus orígenes, el estudio de los negocios ha buscado incansablemente ser parte de aquellas disciplinas consideradas "duras". Desde que la Americanización (Juusola, Kettunen, \& Alajoutsijärvi, 2015) alcanzó a las escuelas de negocios en Chile, estas han profundizado su compromiso con teorías y metodologías reduccionistas que no aportan mucho al entendimiento de los fenómenos organizacionales, y aún menos a la reflexión moral sobre los mismos (Ghoshal, 2005; Abend, 2014; Anteby, 2013), pero que sí permiten "ponerle números" a las cosas. El ejemplo de Chile y su tozudez por revestir el estudio de lo comercial con la capa de la ingeniería es, quizá, la mejor muestra de aquel deseo 


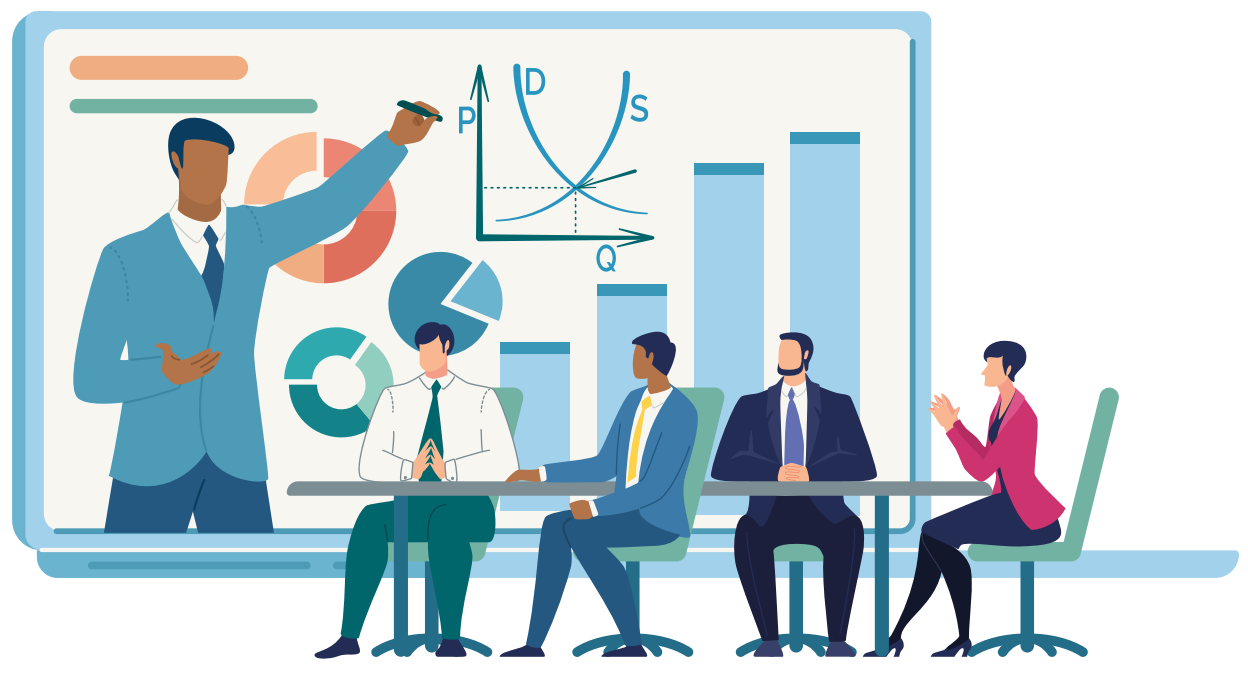

"Afortunadamente, y como suele suceder con los cambios de paradigma, a veces llega un puntapié inesperado que acelera exponencialmente la velocidad de éste. Ese puntapié llegó"

perenne por sumergirse en las aguas de la objetividad.

La aspiración de ser considerada una ciencia exacta proviene de las críticas que despertó la creación de escuelas de negocios, a las que se les acusaba de no ser ni suficientemente prácticas ("no se puede enseñar a administrar en una sala de clases") ni tampoco suficientemente teóricas ("esto ni siquiera es ciencia"). El debate conocido en la literatura como la brecha rigor-relevancia (Syed, Mingers, \& Murray, 2010) pone en tensión dos posturas que, hasta el día de hoy, se encuentran presentes en cada escuela de negocios. La postura del rigor sostiene que la educación en negocios debiese emanar de la investigación académica de punta, y preferentemente de aquella respaldada por modelamientos estadísticos, sin importar si lo que se enseña provee utilidad práctica alguna a los futuros profesionales. Por otro lado, quienes defienden la postura de la relevancia sostienen que la educación en negocios debiese entregar herramientas a sus estudiantes con el fin de mejorar sus habilidades prácticas, sin importar si éstas emanan de sofisticaciones intelectuales o no.

Hoy en día, sin embargo, las escuelas de negocios han escindido la búsqueda del rigor y la relevancia en distintas áreas. Por el aporte público que el contenido de la investigación genere no es ni de cerca tan importante como su universalización (Flores \& Céspedes, 2020). En el estudio de lo organizacional, esto conlleva a enfocarse en indigeribles sofisticaciones matemáticas y en un fetiche de los grandes números -encuestas- que permiten hacer calificar una investigación como algo de interés independientemente de lo que ésta se trate. Los incentivos -bonos y premios- están modelados a través de estos parámetros puesto que les permiten a las escuelas el escalar en rankings como el QS o el THE. En resumen, el rigor se ha resumido en la incansable publicación de investigación que muchas veces no genera ningún beneficio al país ni posee conexión alguna con lo enseñado a los estudiantes, pero que le permiten a la institución y al académico posicionarse internacionalmente.

\section{Un modelo exitoso, pero ¿Hasta cuándo?}

Este modelo de desarrollo ha permitido a las escuelas de negocios lograr una expansión exponencial durante las últimas décadas. Como botón de muestra, el año pasado se titularon en Chile 6.943 estudiantes de Ingeniería Comercial. Si sumamos carreras afines como Control de Gestión, Contador Auditor, y todas las variaciones técnicas de estos grados, la cifra asciende a casi 20.000. Esto representa un $8 \%$ del total de titulados del país en el último año (Valenzuela, 2020). El modelo parece infalible; mientras investigadores proveen prestigio institucional, "profes taxi" satisfacen los deseos de estudiantes-clientes dispuestos a pagar por aumentar su empleabilidad.

Sin embargo, y al igual que pasa con cualquier otro producto, la masificación de la educación en negocios y su direccionamiento a través del mercado laboral la han empujado hacia la estandarización. Hoy resulta imposible diferenciar a un profesional de negocios de, por ejemplo, una universidad confesional y privada, de otro de una universidad laica y pública. Pero, si la influencia de las empresas ha reducido las mallas curriculares a un cúmulo de saberes prácticos comunes, entonces ¿qué diferencia a dos escuelas de negocios? Esta pregunta, durante las últimas décadas al menos, ha sido respondida a través de los procesos de socialización que ejercen los espacios 
educativos (Ehrensal, 2001; Vaara \& Faÿ, 2012). Al tener estudiantes provenientes de entornos con distintos capitales sociales y culturales, se espera que la interacción entre ambos mundos permita generar redes de contacto que faciliten tanto la movilidad social como la cohesión. La composición relativa de cada escuela es, en gran medida, la razón por la cual se escoge una u otra institución. Sin embargo, en un contexto de educación remota donde las interacciones se dan de manera online, este argumento pierde la mayor parte de su peso. Si ahora el estudiantado se reduce a una serie de pantallas negras con voces en off, ¿En qué se basará el estudiante-cliente al momento de elegir dónde matricularse?

Más aún, si la educación online ha llegado para quedarse como sugieren los primeros indicios dados por instituciones que ya se comprometieron a un 2021 online, ¿Qué le impide al estudiante-cliente elegir una institución extranjera mejor posicionada que cualquiera de las escuelas de negocios chilenas disponibles? Incluso se podría argumentar bajo el prisma de la socialización que esta elección implicaría el estar expuesto a capitales culturales y sociales aún más diversos y, por ende, con mejor potencial de generar redes de contacto y emprendimientos en un mundo ya altamente globalizado.

Me permito ir incluso más lejos con el cuestionamiento; con la educación en negocios transformada en un commodity, ¿Tiene siquiera algún sentido el matricularse en una escuela de negocios cuando la mayor parte de aquellos contenidos ya se encuentran disponibles de manera gratuita en plataformas digitales? Nuevamente, durante las últimas décadas, esta pregunta era fácilmente respondida a través de la función acreditadora de la educación superior (Brown, 2001), es decir, la creencia legitimada socialmente de que una persona se encuentra calificada por haber cursado de forma exitosa una carrera en una institución educativa formal. La función acreditadora de la educación superior sirve como un mecanismo señalizador de los "filtros" que el estudiante tuvo que enfrentar para convertirse en profesional. A mayor prestigio de la institución se asumen filtros más estrictos. Este prestigio de las instituciones

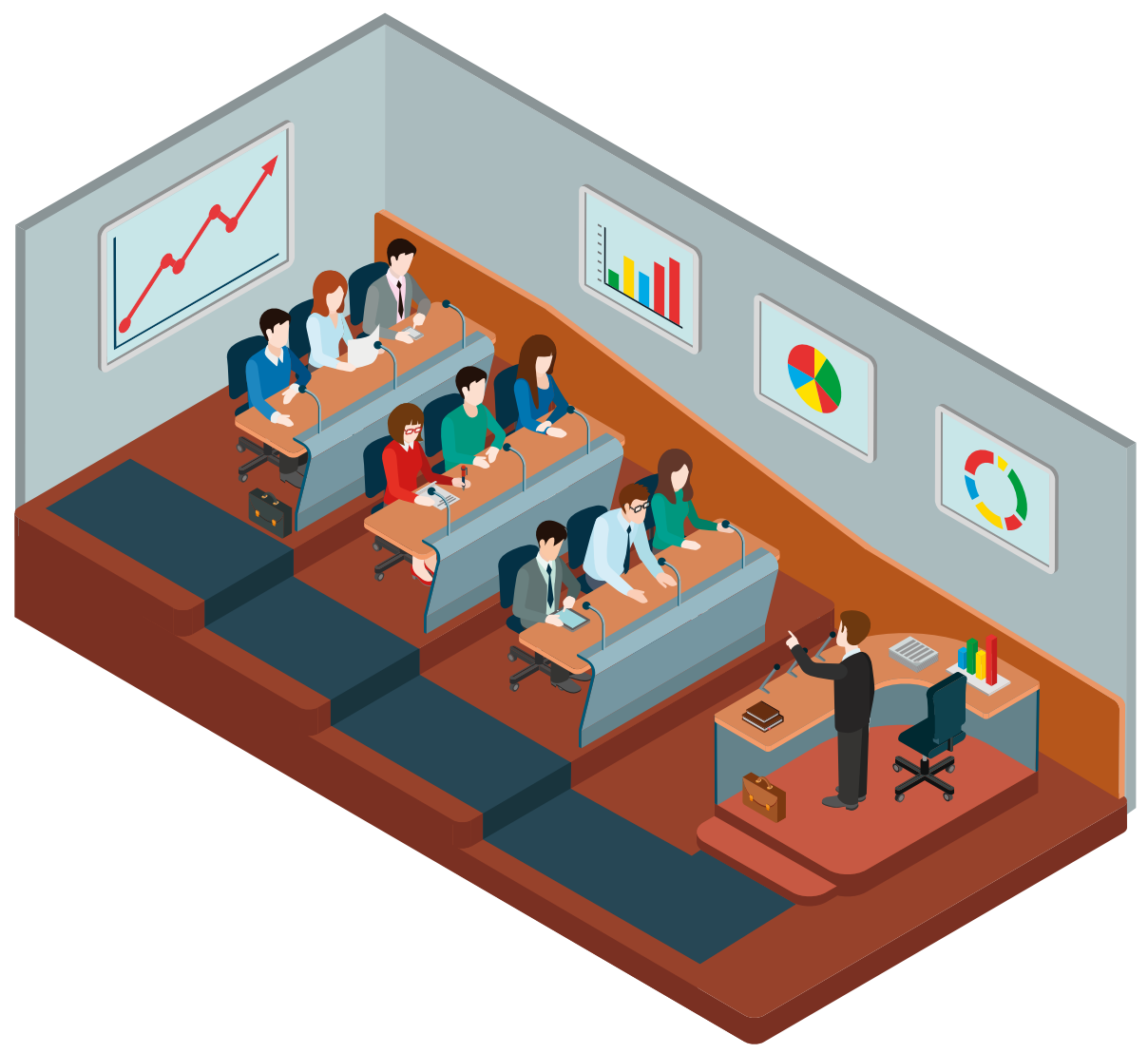

educativas se basa, fundamentalmente, en su capacidad para medir y controlar el aprendizaje obtenido por el estudiante. Sin embargo, en un contexto de educación online, aquel panóptico pierde gran parte de su efectividad predictiva. (¿Sabrán los profesores de las escuelas de negocios que sus alumnos hacen las pruebas online a través de Discord?) Es razonable, entonces, el proyectar que el mercado laboral sabrá leer este cambio en la calidad de la señalización y, al igual como ya lo hacen muchas empresas de tecnología e innovación, confíen cada vez menos en los títulos y más en la experiencia real y comprobable de sus postulantes.

Por último, las carreras de negocios no necesitan laboratorios ni clase alguna de trabajo manual que fuercen la presencialidad, es decir, son parte de las llamadas "carreras de pizarrón". Hoy en día la única justificación que se brinda a los estudiantes para hacerles pagar aranceles medidos en millones de pesos es la provisión de infraestructura como bibliotecas y computadores, los cuales se encuentran en desuso. Resulta razonable también el proyectar que lo seguirán estando en gran medida incluso luego de superada la peor parte de la pandemia. ¿Qué incentivos tienen, entonces, los potenciales estudiantes de negocios para adquirir una pesada mochila de deuda a cambio de una credencial que tendrá progresivamente menos peso al momento de evaluar su empleabilidad? Sin duda, el financiar investigación desvinculada de la educación que reciben no es uno de ellos.

\section{Lo humano como problema}

La propuesta de valor de las escuelas de negocios ha sido extremadamente exitosa durante décadas, pero su relación con el mercado también ha generado un "amarre de manos"(Khurana, 2007) que le dificulta evolucionar al ritmo que lo hace un entorno impredecible. La transición del paradigma educacional prusiano ${ }^{1}$ hacia un nuevo relacionamiento basado en interacciones digitales era previsible, e incluso estaba siendo empujado de manera interna desde hace ya varios años por académicas y académicos jóvenes. Sin embargo, las jerarquías altamente

(1) Modelo educativo basado en principios de obligatoriedad (el Estado mandata), jerarquización (hay niveles que superar), y estandarización (hay ciertos conocimientos que son los valiosos). 
verticales de la educación superior -y que rozan lo patronal en el caso de las escuelas de negocios- han retrasado este avance durante más de una década. De hecho, las voces solicitando revisar, modernizar, y/o redefinir el rol de las escuelas de negocios comenzaron hace ya muchos años (Alvesson \& Willmott, 1992; French \& Grey, 1996), se han robustecido durante décadas de desarrollo teórico (Alvesson \& Willmott, 2003; Grey, 2004; Steyaert, Beyes, \& Parker, 2016), y han dado paso a una gran diversidad de enfoques; sostenibilidad (Beusch, 2014), responsabilidad (Blasco, 2011), justicia social (Fotaki \& Prasad, 2014; 2015; Toubiana, 2014), ciudadanía global (Lilley, Barker, \& Harris, 2014), humanismo (Von Kimakowitz, Pirson, Spitzeck, Dierksmeier, \& Amann, 2011), e incluso, liberalismo (Harney \& Thomas, 2013). Las escuelas de negocios en Chile, sin embargo, se han mantenido mayormente ajenas a estos desarrollos. Mientras en el mundo la academia de vanguardia sugiere "cerrar" las escuelas de negocios para transformarlas en es- cuelas de organizaciones (Parker, 2018), en Chile quienes dirigen estas instituciones educativas siguen aferrados a los mismos paradigmas que han abrazado desde la guerra fría.

La razón de este conservadurismo, y que a su vez es el mayor obstáculo que tienen las escuelas de negocios para responder a los cambios actuales, es aquel inagotable anhelo de ser ciencia exacta en el que fueron formados sus líderes y el cual se han encargado religiosamente de profundizar durante las últimas décadas. Al concebir el estudio de los negocios como algo que puede ser purificado de las imperfecciones que aportan las personas y posteriormente empaquetado como un producto genérico, se acepta la falacia de que los fenómenos grupales y organizacionales pueden ser conducidos sin dejar espacio a lo impredecible o azaroso (Roberts, 1996; Gabriel, 1998). Esta concepción "ingenieril" de lo organizacional donde todo se puede y debe manejar y controlar les impide entender la función que cumplen las complejidades humanas; el rol que juegan las emociones y los ideales en nuestras decisiones, el potencial creativo que reside en el ocio y en el descanso, o la riqueza que se genera a través del debate que no desemboca en un consenso. Peor aún, su concepción disciplinar de los negocios -y por extensión, de la educación en negocios- no les permite lidiar con las incertidumbres que fluyen de manera subterránea y que, en el momento adecuado, emergen y lo remecen todo.

Afortunadamente, y como suele suceder con los cambios de paradigma, a veces llega un puntapié inesperado que acelera exponencialmente la velocidad de éste. Ese puntapié llegó.

Bibliografía

Abend, G. (2014). The Moral Background: An Inquiry Into the History of Business Ethics. Princeton University Press. Alvesson, M., \& Willmott, H. (1992). Critical Management Studies. London : Sage.

Alvesson, M., \& Willmott, H. (2003). Studying Management Critically. London: Sage.

Anteby, M. (2013). Manufacturing Morals: The Values Of Silence In Business School Education (1st ed.). Illinois: University of Chicago Press.

Bennis, W., \& O’Toole, J. (2005, May). How Business Schools Lost Their Way. Harvard Business Review.

Beusch, P. (2014). Towards sustainable capitalism in the development of higher education business school curricula and management. International Journal of Educational Management, 28(5), 523-545.

Blasco, M. (2011). Aligning the Hidden Curriculum of Management Education With PRME: An Inquiry-Based Framework. Journal of Management Education, $36(3), 364$ - 388. Brown, D. K. (2001). The Social Sources of Educational Credentialism: Status Cultures, Labor Markets, and Organizations. Sociology of Education, 74(Extra Issue), 19-34. Ehrensal, K. N. (2001). Training Capitalism's Foot Soldiers: The Hidden Curriculum of Undergraduate Business Education. In E. Margolis, The Hidden Curriculum in Higher Education (pp. 97-113). Routledge.

Flores, T., \& Céspedes, D. (2020, 02 19). La muerte -no declarada- de la Universidad capitalista. El Desconcierto. Retrieved from https://www.eldesconcierto.cl/2020/02/19/ la-muerte-no-declarada-de-la-universidad-capitalista/

Fotaki, M., \& Prasad, A. (2014). Social justice interrupted? Values, pedagogy, and purpose of business school academics. Management Learning, 45(1), 103-106. Fotaki, M., \& Prasad, A. (2015). Questioning Neoliberal Capitalism and Economic Inequality in Business Schools. Academy of Management Learning \& Education, 14(4), 556575.

French, R., \& Grey, C. (1996). Rethinking Management Education. London: SAGE.

Gabriel, Y. (1998, September). The Hubris of Management. Administrative Theory \& Praxis, 20(3), 257-273.

Ghoshal, S. (2005). Bad Management Theories Are Destroying Good Management Practice. Academy of Management Learning and Education, 4(1), 75-91.

Grey, C. (2004). Reinventing Business Schools: The Contribution of Critical Management Education. Academy of Management Learning and Education, 3(2), 178-186. Harney, S., \& Thomas, H. (2013). Towards a liberal management education. Journal of Management Development, 32(5), 508-524.

Juusola, K., Kettunen, K., \& Alajoutsijärvi, K. (2015). Accelerating the Americanization of Management Education: Five Responses From Business Schools. Journal of Management Inquiry, 24(4), 347-369.

Khurana, R. (2007). From Higher Aims to Hired Hands: The Social Transformation of American Business Schools and the Unfulfilled Promise of Management as a Profession. Princeton: Princeton University Press.

Lilley, K., Barker, M., \& Harris, N. (2014). Educating global citizens in business schools. Journal of International Education in Business, 7(1), $72-84$.

Parker, M. (2018). Shut Down the Business School! An insider's account of what's wrong with management education (1st ed.). London: Pluto Press.

Roberts, J. (1996). Management Education and the Limits of Technical Rationality: The Conditions and Consequences of Management Practice. In R. French, \& C. Grey (Eds.), Rethinking Management Education (pp. 54-75). London: SAGE.

Steyaert, C., Beyes, T., \& Parker, M. (2016). The Companion to Reinventing Management Education. London: Routledge.

Syed, J., Mingers, J., \& Murray, P. A. (2010). Beyond rigour and relevance: A critical realist approach to business education. Management Learning, 41 (1), 71-85.

Toubiana, M. (2014). Business pedagogy for social justice? An exploratory investigation of business faculty perspectives of social justice in business education. Management Learning, 45(1), 81-102.

Vaara, E., \& Faÿ, E. (2012). Reproduction and Change on the Global Scale: A Bourdieusian Perspective on Management Education. Journal of Management Studies, 49(6), 1023-1051.

Valenzuela, Ó. (2020, Septiembre 30). Cuáles son las carreras universitarias con más titulados. Las Últimas Noticias, p. 25. Retrieved from https://www.lun.com/Pages/NewsDetail.aspx?dt=2020-09-30\&Paginald=25\&bodyid=0

Von Kimakowitz, E., Pirson, M., Spitzeck, H., Dierksmeier, C., \& Amann, W. (2011). Humanistic Management in Practice (1st ed.). Basingstoke: Palgrave Macmillan. 